\title{
Surface Waves and Wave Resistance in Magnetic Fluids
}

\author{
J. Browaeys, R. Perzynski*, J.-C. Bacri*, and M. I. Shliomis ${ }^{\S}$ \\ Received on 15 February, 2001
}

\begin{abstract}
We present here the experimental determination of the dispersion relation of surface waves of a ferrofluid submitted to a steady vertical magnetic field. The results are compared to a linear theory where all the magnetic characteristics of the fluid have been taken into account. The knowledge of the dispersion equation is then used to analyse the capillary-wave resistance, that is the drag force associated to the emission of waves by a moving disturbance at a free fluid surface. It undergoes a transition from zero to a finite value as the speed of the disturbance reaches a certain critical value. The effect of viscosity is explored, and a magnetic fluid is shown to allow controlling the critical speeds. Contrary to the theoretical model, however, the measured wave resistance reveals a non monotonic speed dependence after the threshold.
\end{abstract}

\section{Introduction}

Magnetic fluids, sometimes called ferrofluids, are colloidal suspension of magnetic particle which have a giant magnetic susceptibility, usually in the order of unity. When submitted to he influence of an external DC vertical magnetic field, one is stricken by the structuration of its surface into peaks, usually distributed on an quasi-hexagonal array. The linear analysis of this phenomenon [1], called after its discoverer the "Rosensweig instability" has been the foundation stone of ferrohydrodynamics [2], the thermo-hydrodynamics of non-conducting magnetic liquids. The fluid peaks arise because capillary-gravity waves, either induced by thermal fluctuations or ambient vibrations, are amplified when a sufficiently strong vertical magnetic field is present.

The precise knowledge of the dispersion equation is of paramount importance for any process that is sensitive to surface instabilities. In fact, every classical hydrodynamical instability such as the Rayleigh-Taylor, or Bénard-Marangoni instabilities [3], has a magnetic counterpart with ferrofluids. The additional magnetic field, acting as a new control parameter, usually allows to control the threshold of the instability [4].

The first part of this paper deals with the measurement of the dispersion equation of capillary-gravity surface waves of a magnetic fluid under the influence of a vertical and steady magnetic field. The results are con- fronted to a linear theory that takes into account all the magnetic characteristics of the ferrofluid sample used. The results are then used to analyse a threshold phenomenon : the capillary-gravity wave resistance.

When an object is moved at the free surface of a fluid, it experiences a drag force which physically originates from: (a) bulk dissipation in a viscous boundary layer for low Reynolds numbers, and in the turbulent wake for high Reynolds numbers; (b) the emission of capillary-gravity surface waves. Such waves remove momentum from the perturbating object to infinity. The associated force that the object experiences is called wave resistance.

For the convenient moderate speeds on which we focus in this paper it may overcome the bulk dissipation type drag. Wave resistance has been studied for more than a century in the case of pure gravity waves [5], mainly because this topic has obvious naval applications [6]. However the case of capillary-gravity waves has only been theoretically treated in a recent work of Raphaël and de Gennes [7]. The dispersive properties of such waves are such as there exists a minimum phase speed $V_{c}=(4 \sigma g / \rho)^{1 / 4}$ at which waves are able to propagate (here $\sigma$ is the surface tension of the free air-fluid interface and $\rho$ the density of the fluid). Since the wave pattern is stationary in the reference frame of the moving object, no wave can be emitted for $V<V_{c}$ [8], and therefore there is no wave resistance in that case. As modeled in [7], the wave resistance should experience a

\footnotetext{
* Groupe Ferrofluide associé à l'Université Denis Diderot - Laboratoire des milieux désordonnés et hétérogènes, UMR CNRS 7603 Université Pierre et Marie Curie, case 86, 4 place Jussieu 75252 Paris Cedex 05, France.

${ }^{\dagger}$ Laboratoire de Bio-rhéologie et d'Hydrodynamique Physico-chimique, ESA CNRS 7057 Université Denis Diderot, case 7056, 2 place Jussieu 75251 Paris Cedex 05, France.

$\ddagger$ E-mail: browaeys@ccr.jussieu.fr

$\S$ Department of Mechanical Engineering, Ben-Gurion University of the Negev, P.O.B. 653, Beer-Sheva 84105, Israel
} 
finite jump $R_{c}$ at $V=V_{c}$ and increases above $V_{c}$. The system is thus supposed to undergo a discontinuous bifurcation.

The second part of this work deals with the measurement of the wave resistance. In order to check these theoretical predictions it is necessary to vary $V_{c}$. Varying $\rho$ and $\sigma$ is very ineffective way to change $V_{c}$ (it would merely vary from $17 \mathrm{~cm} / \mathrm{s}$ to $23 \mathrm{~cm} / \mathrm{s}$ for any kind of fluid). We here show theoretically and experimentally that the action of a magnetic field on a magnetic fluid provides a means to tune the critical velocity from its maximum value $V_{c}^{H=0}=(4 \sigma g / \rho)^{1 / 4}$ down to 0 .

\section{Surface waves}

\subsection{Dispersion equation}

Cowley \& Rosensweig [1] were the first to study the peak instability at the free surface of a magnetic fluid. Although they didn't actually express the dispersion equation for waves at the free surface of a ferrofluid (they were interested in the threshold value of the magnetic field at which the peaks form), they developed the linear theory which allows to directly derive the magnetic term in the dispersion equation. They considered that the ferrofluid was inviscid, that its depth was infinite and that it was infinitely extended in the horizontal direction.

Since then, other authors refined this analysis including more realistic conditions : Zelazo \& Melcher [9] expressed the dispersion equation of surface waves of a ferrofluid layer resting between the poles of a magnet. More recently, Abou \& al. [10] changed this boundary condition (the layer is generally surrounded by free space, for visualization purposes), and included the effect of viscosity. However, they omitted to take into account the demagnetization coefficient and also supposed that the permeability of the material was constant, a fact that is not supported experimentally, since a magnetization saturation exists in the material. This shortcoming has been removed in an article of the coauthors [11].

In order to simplify, only their main results are presented here. In the case of constant permeability for an inviscid fluid of infinite depth, the dispersion relation writes :

$$
\omega^{2}=g k+\frac{\sigma k^{3}}{\rho}-\frac{\mu_{0} H^{e 2} k^{2}}{\rho}\left(\frac{\chi^{2}}{(1+\chi)(2+\chi)}\right),
$$

where $\omega$ is the pulsation, $k$ the wave vector, $g$ the gravitational acceleration, $\sigma$ the surface tension of the ferrofluid layer, $\rho$ its density, $\mu_{0}$ the vacuum permeability, $H^{e}$ the external vertical magnetic field and $\chi$ the magnetic susceptibility.

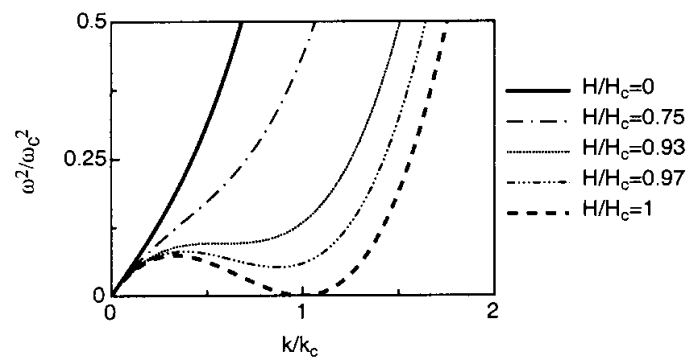

Figure 1. Dispersion relation of surface waves in a ferrofluid. Here $\omega_{c}=2\left(\rho g^{3} / \sigma\right)^{1 / 4}$ is introduced to normalize the curves. It corresponds to the pulsation when $k=k_{c}$ without magnetic field.

The first two terms of the right side of eq. (1) are classical and respectively account for the gravity and capillarity effect. The last term includes the effect of the magnetic field. It is a destabilizing term : at the critical field $H_{c}$, the dispersion curve (Fig. 1) adjoins the zero frequency axis at a non zero wavevector $k_{c}$. Analytical derivation yields :

$$
\begin{aligned}
H_{c} & =\sqrt{\frac{2(1+\chi)(2+\chi)}{\chi^{2}} \frac{\sqrt{\rho g \sigma}}{\mu_{0}}} \\
k_{c} & =\sqrt{\frac{\rho g}{\sigma}}
\end{aligned}
$$

It is noteworthy that the wavevector at the threshold of the Rosensweig instability is indeed the capillary wavevector.

In the more realistic case where the fluid layer has finite depth, is viscous and its magnetic susceptibility is field dependent, the general picture previously presented is only slightly modified.

Including viscosity in the previous equations renders them very complex, and as we show further on, the terms accounting for viscosity in the dispersion equation can most of time be omitted. It is possible to define two Reynolds numbers [10], either based on the depth $h$ of the ferrofluid layer, or based on the wavelength :

$$
R e=\frac{\omega h^{2}}{\nu}, \quad \text { or } \quad R e=\frac{\omega}{\nu k^{2}} .
$$

The influence of viscosity on the flow generated by surface waves is negligible provided the Reynolds number is greater than 10 [12]. This leads to two conditions for the pulsation $\omega$ for short and long wavelengths :

$$
\omega>\sup \left(10 \frac{\nu}{h^{2}}, 10 \nu k^{2}\right) \text {. }
$$

In Figs. 5 and 6, the area of the parameters where the viscosity cannot be neglected (according to the previous equation) has been shaded. Most of the experimental points lie outside this region.

Finally, it is important to note that the experiment is realized in cylindrical geometry : the produced waves are concentric. The derivation of the dispersion equation has however assumed plane waves. As long as the 
observed wavelength is smaller than the radius of the ferrofluid container, it is possible to neglect the effect of geometry. To be more precise, the Bessel $J_{0}$ function is the basis for the cylindrical capillary-gravity wave dispersion equation. If the distance between two consecutive crests is assumed to be the plane-wave wavelength, then the error is $11 \%$ for the first two crests (starting from center), and then only $4 \%$ for the next two (and decreasing as the radius increases). Since the radius of the container is $9.6 \mathrm{~cm}$, the wavelengths are measured in at least within a $11 \%$ approximation if they are smaller than $4.8 \mathrm{~cm}$, which correspond to a limiting wavevector of $1.3 \mathrm{~cm}^{-1}$. The complete calculation of the dispersion equation including the magnetic term in a cylindrical geometry is a theoretical problem that has yet to be solved; it might yield a better understanding of the effect of non-periodic magnetic field perturbations.

\section{I.2 Experimental setup}

\section{I.2.1 Characteristics of the ferrofluid sample}

We use an ionic ferrofluid synthesized according to Massart's method [13]. It is composed of a colloidal suspension of cobalt ferrite particles in water. Its density is $1560 \mathrm{~kg} / \mathrm{m}^{3}$ and the deduced volume fraction of particles is $14 \%$. Its surface tension with air, carefully measured with a Krüss K10T ring tensiometer, is $71.4 \mathrm{mN} / \mathrm{m}$ at $20 \mathrm{C}$. This value is very close to the surface tension of water $(73.2 \mathrm{mN} / \mathrm{m})$, in agreement with the fact that our ionic ferrofluid is free of surfactant. The dynamic viscosity of the sample, measured with a Poiseuille viscometer, is $20 \mathrm{mPa} . \mathrm{s}$ which leads to a kinematic viscosity of $1.2810^{-5} \mathrm{~m}^{2} / \mathrm{s}$.

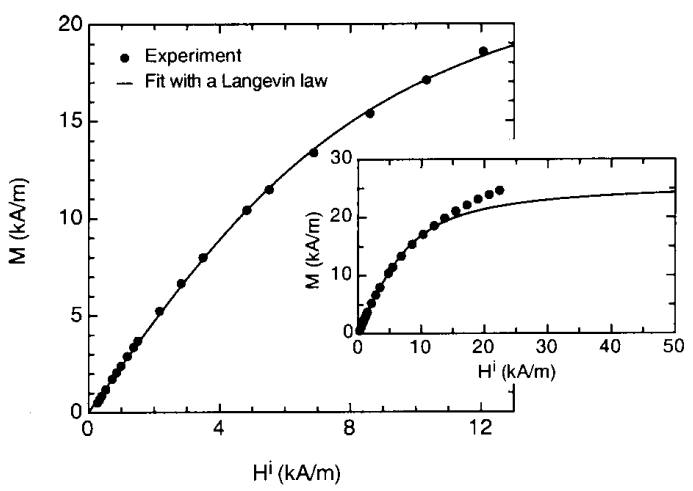

Figure 2. Magnetization curve of the ferrofluid sample. The best least square fit with equaton (6) gives $A_{0}=26.15 \mathrm{kA} / \mathrm{m}$ and $A_{1}=3.63 \mathrm{kA} / \mathrm{m}$. inset : same curves for a more extended magnetic field range. The Langevin curve does not fit the whole magnetizaion curve.

The magnetization curve has been obtained by the use of a calibrated fluxmeter (Fig. 2). Because our ferrofluid is highly concentrated and polydisperse, it is unlikely to follow a classical Langevin paramagnetic curve. However, this approximation may stand for the range of magnetic field amplitude of our interest (from 0 to about $12 \mathrm{kA} / \mathrm{m}$, the field around which the peaks instability develops) :

$$
M \approx A_{0}\left(\operatorname{coth}\left(H^{i} / A_{1}\right)-A_{1} / H^{i}\right)
$$

Here $M$ is the magnetization and $H^{i}$ is the magnetic field inside the ferrofluid : $H^{e}=H^{i}+M$. It should be noted that the $A_{0}$ value obtained is a priori different from the magnetization saturation of the ferrofluid sample. The knowledge of the parameters of the fit $A_{0}$ and $A_{1}$ allows us to compute all the magnetic parameters of the problem.

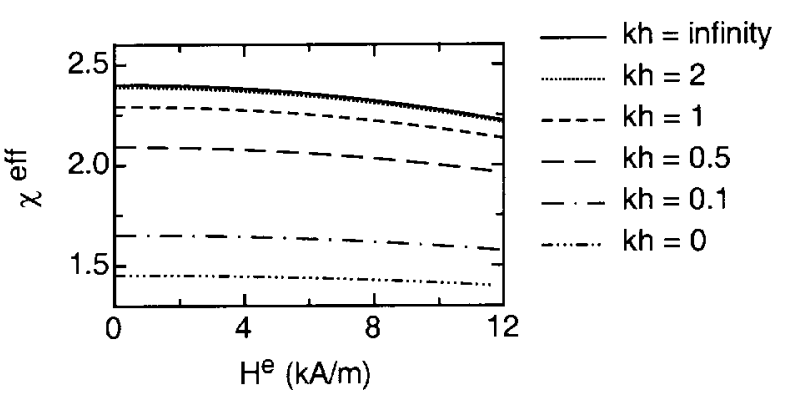

Figure 3. Effective magnetic susceptibility $\chi_{\text {effas }}$ a function of the external magnetic field $H^{e}$ for differnet nondimensinal wavevectors $k h$.

Eq. (1) represents the dispersion equation when magnetic permeability is a constant, which is obviously not the case here. Following [11], it is possible to define an effective magnetic susceptibility $\chi_{\mathrm{eff}}$, function of the magnetic field and the non-dimensional product $k h$, and computed so that eq. (1) is correct. It is also a measurement of the discrepancy when the magnetic term of eq. (1) is misused. The following plot (Fig. 3) shows that the value of $\chi_{\text {eff }}$ is almost independent of the magnetic field thus justifying a posteriori the use of a simplified magnetic term (i.e. $\chi$ constant) ; on the other hand it shows that the dependence of the magnetic term on the thickness of the layer has to be accounted for.

\section{I.2.2 Experiment design}

A circular Teflon dish of $20 \mathrm{~cm}$ in diameter that contains the ferrofluid is placed between two horizontal coils; they produce the static vertical magnetic field and are arranged such as to ensure a horizontal spatial homogeneity more accurate than $99 \%$. It should be pointed that the maximum observable wavelength is of same order as the radius of the vessel. 


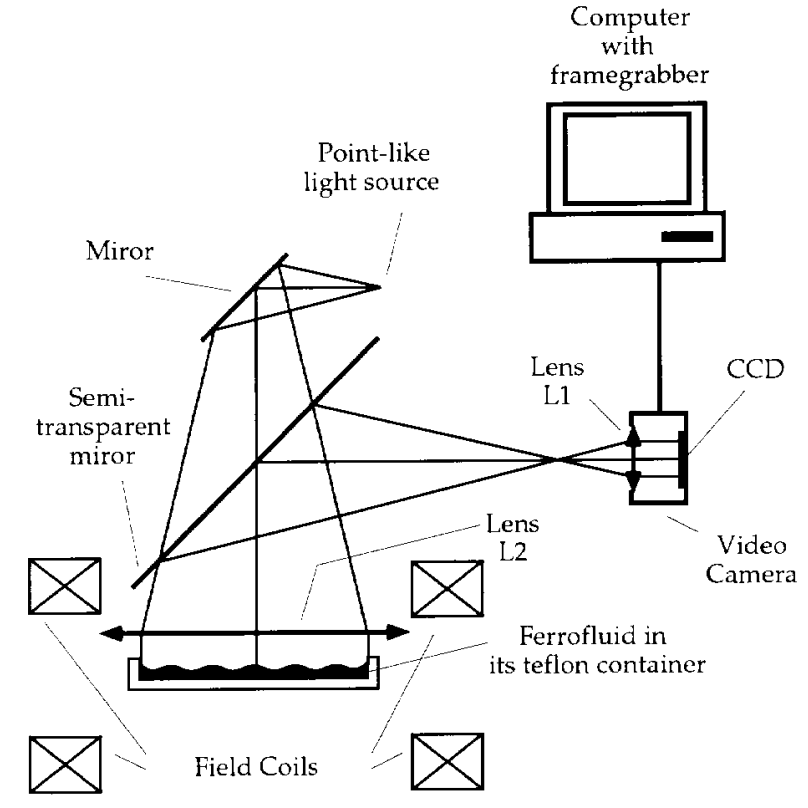

Figure 4. Shadowgraph method.

The shadowgraph method [14] is a very sensitive optical method to detect the wave amplitude $(60 \mu \mathrm{m}$ amplitude ripples are still visible). The ferrofluid surface is illuminated by a parallel light beam coming from a point-like source (an optical fiber illuminator) at the focal distance of lens L1. The camera lens L2 is placed in order to provide a parallel light beam to the CCD detector (see Fig. 4). The distance d between the CCD and the camera lens is set such as to focus just beneath or above the ferrofluid surface. The curved parts of the surface act as virtual lenses, and appear lightened or darkened on the video screen whether the curvature is positive or negative.

To produce surface waves, a small sinusoidal modulation in the vertical field is applied. Propagative circular waves are emitted by the edges due to magnetic forces in the meniscus. Because of the viscous damping [15], no stationary waves are observed, except in the center of the ferrofluid container.

This method is very reliable for the production of waves in the interesting frequency range $(3 \mathrm{~Hz}-$ $25 \mathrm{~Hz}$ ). The undulating component is merely $0.5 \%$ of the threshold field $H_{c}$. It is however difficult to extrapolate the results at fields lower than $0.1 H_{c}$ where the undulation ratio is already $5 \%$.

\section{I.3 Results}

In order to represent experimental results and to fit parameters of the theoretical wave dispersion equation, we chose two cuts in the $\left(k, \omega, \mu_{0} H^{2}\right)$ space of parameters (Fig. 5 and Fig. 6). The only parameter allowed to vary is the surface tension, because of its sensitivity to contaminants. All other parameters (the magnetic quantities, the density and the thickness of the ferrofluid layer) are set to their previously measured values. We have drawn on both Fig. 5 and Fig. 6 the parameter domain where the effects of viscosity could not be neglected: our experimental points lie outside of this zone.

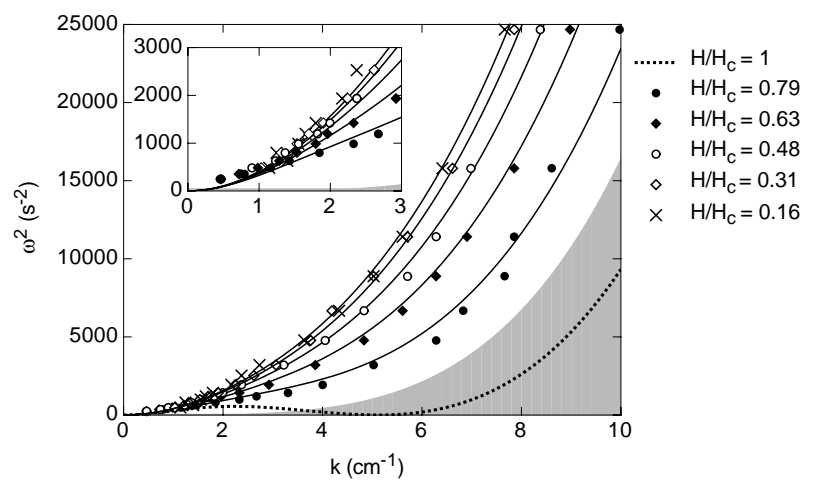

Figure 5. Experimental dispersion equation in the $\left(k, \omega^{2}\right)$ plane for different magnetic fields. The black lines represent the theoretical dispersion equations with $\sigma=60 \mathrm{mN} / \mathrm{m}$. The dotted line is the theoretical dispersion equation at the threshold field. The shaded area represents the frequency domain where viscosity effects become non negligible $(R e<10)$.

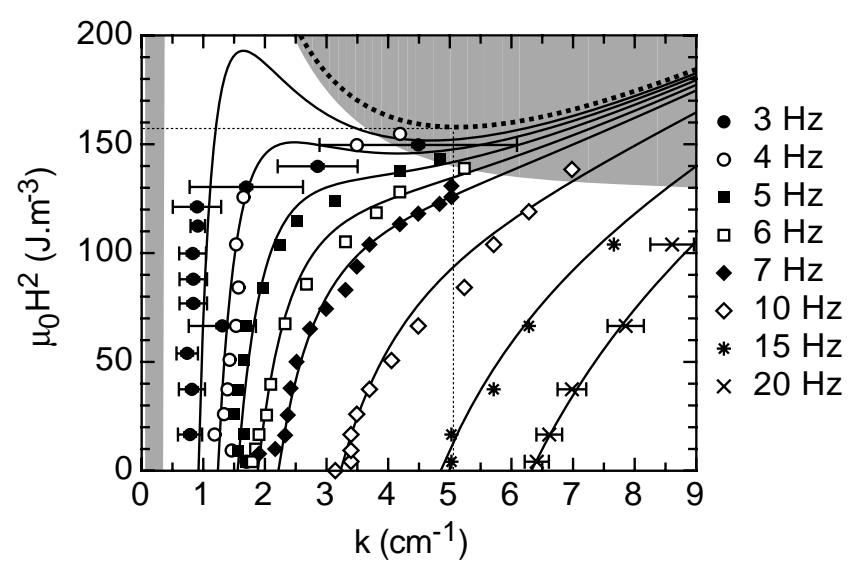

Figure 6. Dispersion equation in the $\left(k, \mu_{0} H^{2}\right)$ plane for different frequencies. The black lines represent the theoretical dispersion equation with $\sigma=60 \mathrm{mN} / \mathrm{m}$; the thick dotted line is the theoretical marginal curve of stability (null frequency); the thin dotted lines locate the minimum of the marginal curve and read $k_{c}=5.1 \mathrm{~cm}^{-1}$ and $\mathrm{HC}=11.2 \mathrm{kA} / \mathrm{m}$. The shaded area represents the frequency domain where viscosity effects become non negligible $R e<10)$. For clarity purpose, only a few error bars have been plotted.

The fit for any value of $\omega$ and $H$ leads to an average surface tension of $60 \mathrm{mN} / \mathrm{m}$, which is $16 \%$ lower than the carefully measured value obtained from a ring tensiometer. The natural contamination of the surface by atmospheric dust behaving as a surfactant may explain this discrepancy. We thus perform another tensiometer measurement by letting the ferrofluid surface rest in an unpurified atmosphere: after a few minutes, the measured surface tension is only $52 \mathrm{mN} / \mathrm{m}$. Since the surface tension is extremely sensitive on the presence 
of any contaminant, the fitted value has to be understood as an in situ value, compatible with tensiometer measurements.

In a first experiment the magnetic field is set to different values and the wavevector measured as a function of frequency. The experimental dispersion is presented in Fig. 5. The fits are satisfactory, although it is difficult to make any assertion when the wavevector is smaller than $3 \mathrm{~cm}^{-1}$. The critical field is estimated from the fits: as we will see later on, it is extremely difficult to define a precise value of the threshold magnetic field $H_{c}$ in our particular system. The maximum experimented value of the ratio $H / H_{c}$ is voluntarily limited to 0.79 because above this field, peaks form at the boundary of the ferrofluid vessel; during the time of the experiment, the fluid in the peaks would dry and become lumpy.

This is why it is better to set the frequency to different fixed values, and measure the wavelength as a function of the magnetic field. The corresponding results are presented in Fig. 6. This plot is more discriminating, especially in the low wavelength domain $\left(k<3 \mathrm{~cm}^{-1}\right)$. Here it can be seen that the fit is not compatible with the experimental data especially in the region where the dispersion curve is non monotonic.

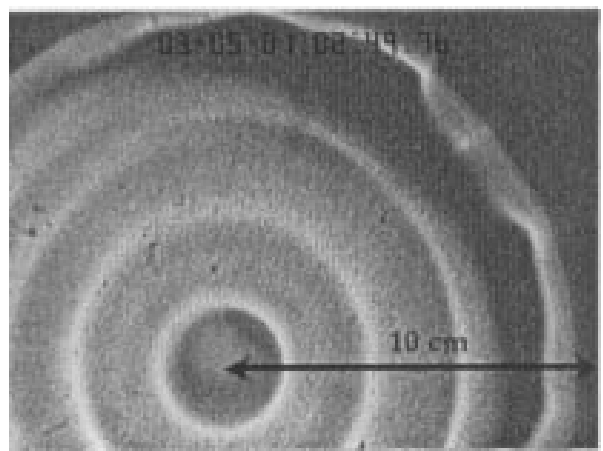

Figure 7. Spontaneous static deformation below the critical field $\left(H / H_{c}=0.79\right)$.

In this region, where the magnetic field is close to $H_{c}$, it seems that an unexpected static circular pattern develops (Fig. 7). It actually becomes visible (amplitude of the deformation exceeds $60 \mu \mathrm{m}$ ) as soon as the magnetic field exceeds half of the threshold value. Only the precision of the shadowgraph method allows to see such a deformation of the surface. The pattern is independent of the magnetic field, only the amplitude of the deformation increases with the magnetic field intensity. It could be understood as a pre-transitionnal effect of the Rosensweig instability, perhaps due to the limited horizontal extension of the layer.

Unfortunately it renders the measurement of the wavelength more uncertain, especially close to the threshold: that is the drawback of the shadowgraph method. It is difficult to precisely assess the value of the threshold magnetic field $H_{c}$. Besides, a experiment with direct visualization of the surface shows that the peaks don't appear simultaneously on the surface : when the field is increased to the threshold value, the peaks grow inward from the edges to the center of the cell (they start appearing on the edges at lower fields, because of the field gradient created by the presence of an edge). A precise estimation of the threshold magnetic field is dependent on the choice of a threshold criteria. We choose to keep the value of the magnetic field above which no image is visible anymore. This method provides a measurement of the threshold field of $11.5 \mathrm{kA} / \mathrm{m}$ which is merely $3 \%$ above the expected value obtained from the fits in Fig. 5 and Fig. 6.

The now precise knowledge of the dispersion equation of surface waves in a ferrofluid submitted to a steady vertical magnetic field allows us to forecast the influence of this magnetic field in other hydrodynamic processes that involve surface waves.

\section{Wave resistance}

\section{II.1 Ordinary fluids}

\section{II.1.1 Theory}

In a regular fluid, the wave emission process is controlled by the dispersion equation for capillary-gravity surface waves,

$$
\omega^{2}=g k+\sigma k^{3} / \rho
$$

where $\omega$ is the pulsation and $k$ the modulus of the wave vector. As already pointed out by Kelvin [5], the wave pattern is stationary in the frame of reference of the moving object.

The dispersion relation of surface waves is valid in the frame of reference $K$ in which the fluid is at rest, and all physical variables (speed, pressure,...) are proportionnal to $\exp (i(\mathbf{k . r}-\omega t))$. In the frame of reference $K^{\prime}$ which translates at speed $\mathbf{V}$, those variables are then proportional to $\exp \left(i\left(\mathbf{k} . \mathbf{r}^{\prime}-\omega^{\prime} t\right)\right)$ where $\mathbf{r}^{\prime}=\mathrm{r}-\mathbf{V} t$ is the position vector in $K^{\prime}$ and $\omega^{\prime}=\omega-\mathbf{k V}$ the pulsation in $K^{\prime}$ (one recognizes the Doppler effect). The stationarity condition implies $\omega^{\prime}=0$ and thus:

$$
\omega=k V \cos \theta
$$

where $\theta$ is the angle between the speed and the wave vector.

Combining (7) and (8) we obtain the following equation $\left(V_{c}=(4 \sigma g / \rho)^{1 / 4}\right.$ the minimum phase speed):

$$
\left(\frac{k}{k_{c}}\right)^{2}-2\left(\frac{V}{V_{c}} \cos \theta\right)^{2}\left(\frac{k}{k_{c}}\right)+1=0,
$$

which has no solution for $V<V_{c}$. Thus no wave pattern is to develop if the speed of the moving perturbation is smaller than $V_{c}$. 
For a Dirac Delta pressure distribution $P(x, y, t)=$ $p \delta(x-V t, y)$ moving along axis $x$, the wave resistance is $[7]$ :

$$
R=\frac{p^{2}}{\pi \sigma} \int_{0}^{\arccos \frac{V_{c}}{v}} \cos \theta \frac{k_{+}(\theta)^{2}+k_{-}(\theta)^{2}}{k_{+}(\theta)-k_{-}(\theta)} \mathrm{d} \theta
$$

where $k_{+}(\theta)$ and $k_{-}(\theta)$ are the two roots of eq. (9). This formula remains valid as long as the characteristic size of the pressure distribution in experiments is much smaller than the capillary wavelength. In those conditions, according to eq. (10), the wave resistance takes a finite value $R_{c}=p^{2} k_{c} / 2 \sqrt{2} \sigma$ just above the threshold and increases monotonically with speed (see the full line in Fig. 12 and the uppermost curve in Fig. 15).

\section{II.1.2 Experiments}

In order to measure $R$ as a function of speed $V$ for various fluids, we use a circular channel dug into a Teflon covered aluminum dish. The latter is fixed to a shaft and rotated at constant rate, thus simulating a steady flow for the fluid. The radius of the channel is $20 \mathrm{~cm}$, its width is $2 \mathrm{~cm}$.

The disturbing object consists of a vertical bronze wire (diameter $d=0.2 \mathrm{~mm}$ ) moving over the fluid surface. The wire is wetted by a few tenths of millimeters of fluid. The deflection of the wire is proportional to the horizontal force exerted on its free end (which is typically in the order of a micronewton). It is measured with an infrared optical sensor (Fig.9). The calibration of the sensor is obtained by tilting the base to which the wire is attached.

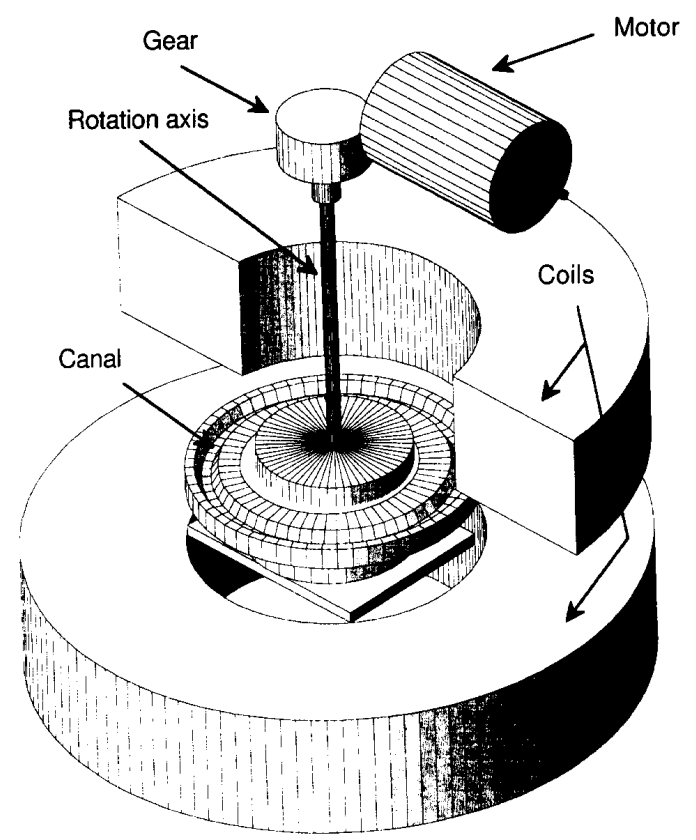

Figure 8. Wave resistance measurement setup : fluid flow system.

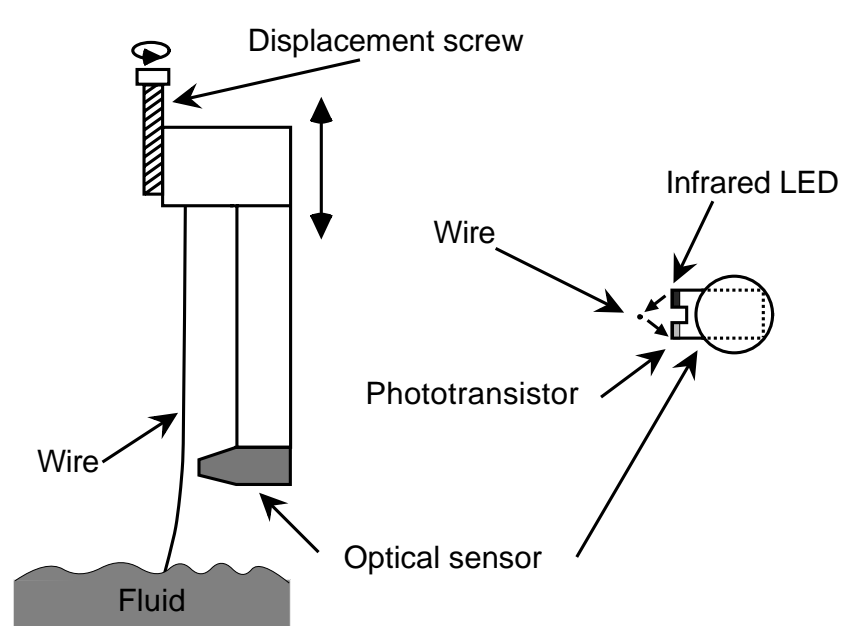

Figure 9. Wave resistance measurement setup: force measurement. The light emitted by the LED is reflected by the wire and collected into the phototransistor.

Because no theory includes $3 \mathrm{D}$ viscous effects so far, we measure the wave resistance for different viscosities. To this purpose several mixtures of water and glycerol are used: the surface tension $\sigma$ and the densities $\rho$ of the mixtures are very close to one another (see table 1 ) so that the impact of viscosity alone may be monitored in our experiments. The viscosities are measured with a standard Poiseuille viscometer.

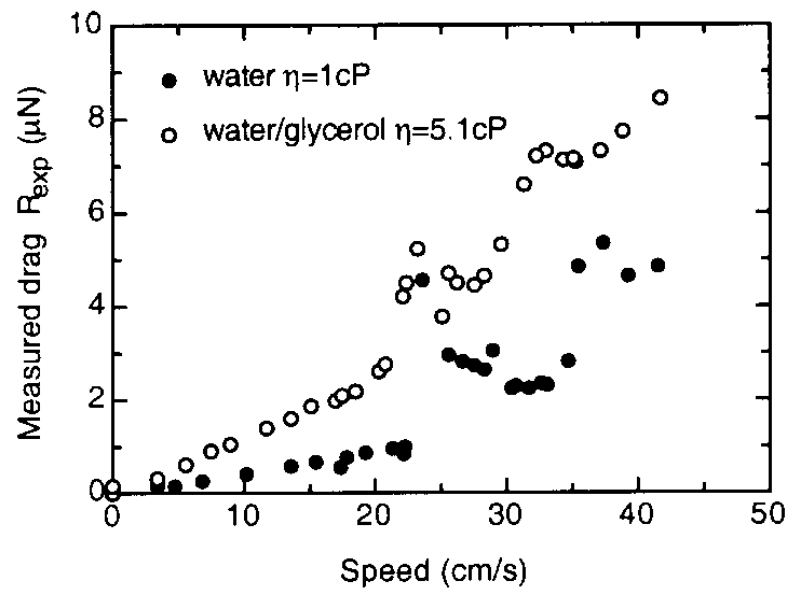

Figure 10. Experimental drag $R_{\text {exp }}$ as a function of speed $V$ for two water-glycerol mixtures.

Fig. 10 displays the variation of the experimental $\operatorname{drag} R_{\text {exp }}$ as a function of speed for two typical mixtures. All the measurements are obtained by increasing and then decreasing the speed: there is no hysteresis. We may note that:

(a) There is a critical velocity at which the measured drag drastically increases. Camera imaging of the fluid surface shows that the sharp drag increase occurs at the same speed at which the wave pattern develops. The measured wavelength is equal to the capillary wavelength (within $10 \%$ error bars). The measured critical 
velocity $V_{c}$ is $23 \pm 0.5 \mathrm{~cm} / \mathrm{s}$ for pure water. It corresponds to a surface tension interval of $65.1-77.7 \mathrm{mN} / \mathrm{m}$ into which lies the tabulated value of pure water surface tension $72.75 \mathrm{mN} / \mathrm{m}$ at $20{ }^{\circ} \mathrm{C}$. For water/glycerol mixtures we obtain $V_{c} \approx 22.5 \mathrm{~cm} / \mathrm{s}$, that is compatible within experimental error bars with the surface tension of the mixtures (around $70 \mathrm{mN} / \mathrm{m}$ ).

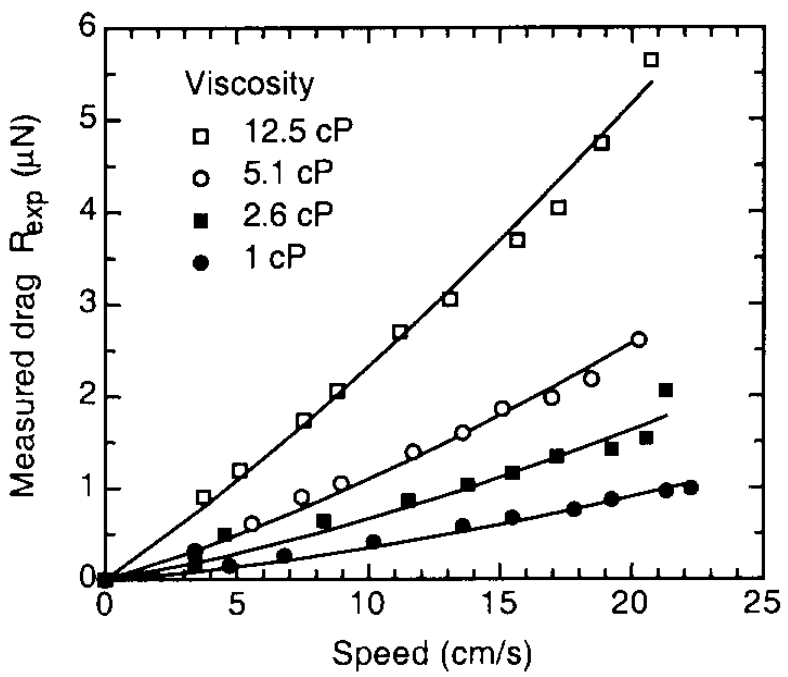

Figure 11. Experimental drag $R_{e x p}$ as a function of speed $V$ for different water-glycerol mixtures. The speed remains subcritical $\left(V<V_{c}\right)$ and only the viscous contribution to the total drag is measured. The plain line represents the phenomenological theory (eg. eq. 11).

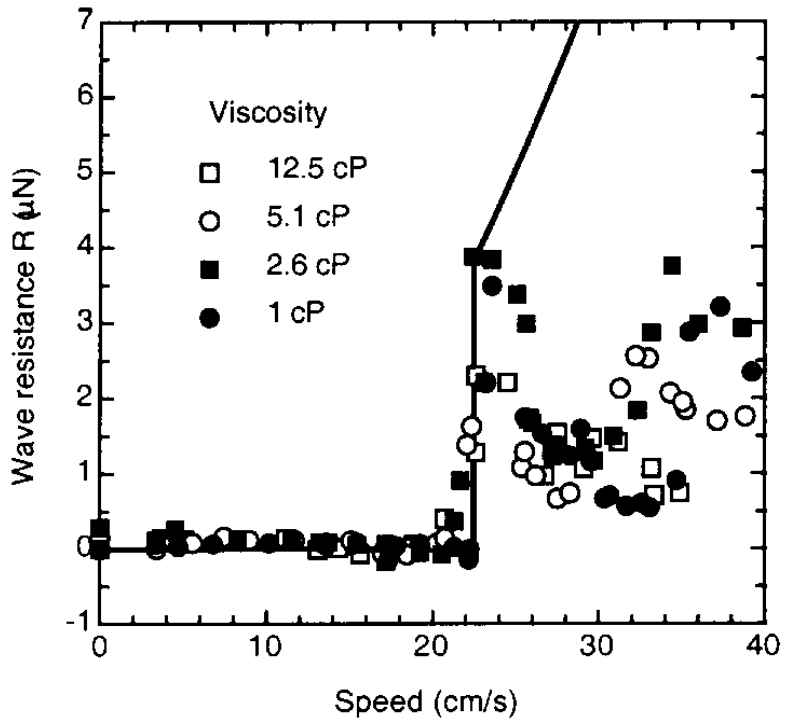

Figure 12. Wave resistance $R=R_{\text {exp }}-R_{v i s c}$ as a function of $V$. Full line : theoretical expression from eq. 10 .

(b) The experimental drag is not null below the critical velocity, all the more since the viscosity is high. The viscous drag $R_{\text {visc }}$ that is exerted over the immersed wire must be added to the wave resistance $R$ to account for the measured drag $R_{\text {exp }}$. Since the length of the wetted part of the wire is comparable to its diameter, we make the assumption that the viscous drag is proportional to that of a sphere of the same diameter $d$. For the moderate Reynolds numbers of our experiment $(0.5<R e<80)$ the viscous drag may be described by an empirical formula [16]:

$$
R_{v i s c}=3 \pi \alpha \eta V d\left(1+0.15\left(\frac{\rho V d}{\eta}\right)^{0.687}\right)
$$

based on numerous sphere drag experiments. In the previous expression $\eta$ represents the viscosity of the solution, $\rho$ its density and $\alpha$ the aspect ratio introduced by us to make allowance for a distinction of our short cylinder from a sphere (for the latter, $\alpha=1$ [16]). A simultaneous fitting procedure on the four $R_{\text {exp }}$ curves for subcritical speeds $(V<18 \mathrm{~cm} / \mathrm{s})$ yields $\alpha=0.77$ (see Fig. 11). Fig. 12 presents the $R(V)$ variations after subtraction of the viscous drag $R_{v i s c}$ for each sample. It is this quantity that has to be compared with the theoretical expression (10) (full line). It seems that a pretransitional effect takes place, as the measured drag increases just below the threshold (the higher the viscosity, the stronger the effect). A recent model [17] for $2 \mathrm{D}$ viscous wave resistance predicts such a feature.

(c) The amplitude of the wave resistance increase at $V_{c}$ is comparable to the theory. Assuming a perfect wetting of the wire by the fluid, the total force acting on the fluid is $p=2 \pi r \sigma$ ( $r$ is the radius of the wire). Thus an estimate of the wave resistance increase at the threshold is given by $R_{c}=\pi^{2} r^{2} \sqrt{2 \rho g \sigma}$. A comparison between expected values and what is observed is given in table 1 . The discrepancy is partially due to the imperfect wetting of the fluid on the wire, which leads to overestimate the applied vertical force. On the other hand the drag values close to the threshold fluctuate a lot.

(d) The wave resistance is a non-monotonic function of speed for $V>V_{c}$. In fact, it can be seen in Fig. 12 that for $V>V_{c}$ the wave resistance $R$ first $d e$ creases as the speed increases, and then increases again for high enough speeds. This feature is not predicted by the current theory, which anyway overestimates the actual drag. Viscosity does not seem to influence the wave resistance decrease. One could also imagine that as the wave pattern develops, the immersion depth of the wire could drop, thus reducing the viscous drag. This is unlikely to occur since we do not observe any scaling of the drag reduction with viscosity. Such a non-monotonicity is possibly a general feature of capillary-gravity wave resistance, and in this case the theory should be revised to include non-linear aspects. 
Table 1: Experimental drag discontinuity at the threshold compared to the theoretical predictions of [4], for various water glycerol mixtures and an aqueous magnetic fluid (MF).

\begin{tabular}{lccccc}
\hline \hline Glycerol mass fraction (\%) & 60 & 44.5 & 30 & 0 & MF \\
\hline Viscosity $(\mathrm{mPa} . \mathrm{s})$ & 12.5 & 5.1 & 2.6 & 1.0 & 7.0 \\
Density $\left(\mathrm{g} / \mathrm{cm}^{3}\right)$ & 1.16 & 1.13 & 1.09 & 1.00 & 1.56 \\
\hline Theory $(\mu N)$ & 3.9 & 3.8 & 3.8 & 3.7 & 4.2 \\
Experience $(\mu N)$ & 2.9 & 2.6 & 4.0 & 3.6 & 4.0 \\
Uncertainty $(\mu N)$ & 0.3 & 0.3 & 0.4 & 1.8 & 1.0 \\
\hline \hline
\end{tabular}

\section{Magnetic fluids}

In a magnetic fluid the dispersion equation of capillarygravity surface waves is modified with allowance for a vertical uniform magnetic field, as shown in eq. (1). For a given wave vector, an increase of the field intensity lowers the frequency of the waves. The frequency drops to zero when $H$ reaches the critical value $H_{c}$ for which the Rosensweig instability is triggered.

The condition for stationarity implies that $k$ must be a solution of:

$$
\left(\frac{k}{k_{c}}\right)^{2}-2\left(\left(\frac{V}{V_{c}} \cos \theta\right)^{2}+\left(\frac{H}{H_{*}}\right)^{2}\right)\left(\frac{k}{k_{c}}\right)+1=0
$$

Real solutions exists if and only if [18]:

$$
V>V_{c}^{H} \quad \text { with } \quad V_{c}^{H}=V_{c} \sqrt{1-\left(\frac{H}{H_{*}}\right)^{2}}
$$

therefore a steady vertical magnetic field should allow the tuning of the critical velocity at which waves (and wave resistance) appear.

The wave resistance, following eq. (10) and eq. (12), is given by the integral:

$$
\begin{gathered}
R^{H}(V)=\frac{p^{2} k_{c}}{\pi \sigma} \int_{0}^{\arccos \left(V_{c}^{H} / V\right)} \cos \theta\left(2 B(V, \theta)^{\frac{1}{2}}+B(V, \theta)^{-\frac{1}{2}}\right) \mathrm{d} \theta \\
\text { where } B(V, \theta)=\left(\left(\frac{V}{V_{c}} \cos \theta\right)^{2}+\left(\frac{H}{H_{*}}\right)^{2}\right)^{2}-1
\end{gathered}
$$

Just above the threshold, the wave resistance has the finite value :

$$
R_{c}^{H}=\frac{p^{2} k_{c}}{2 \sqrt{2} \sigma}\left(1-\left(\frac{H}{H_{*}}\right)^{2}\right)^{-\frac{1}{2}}
$$

An experiment is conducted using a water based magnetic fluid synthesized according to the Massart method [13]. Its critical field $H_{*}$ is $9.15 \mathrm{kA} / \mathrm{m}$ and its surface tension of $60 \mathrm{mN} / \mathrm{m}$ doesn't depend on the magnetic field. Other caracteristics are given in table 1.

The critical value $V_{c}^{H}$ is experimentally estimated as the velocity for which a sudden increase of the drag is observed. $R_{c}^{H}$ is the amplitude of such an increase. Both are plotted versus the normalized magnetic field $H / H_{*}$ in Fig. 13 and Fig. 14, and are compared to theoretical predictions (13) and (16).

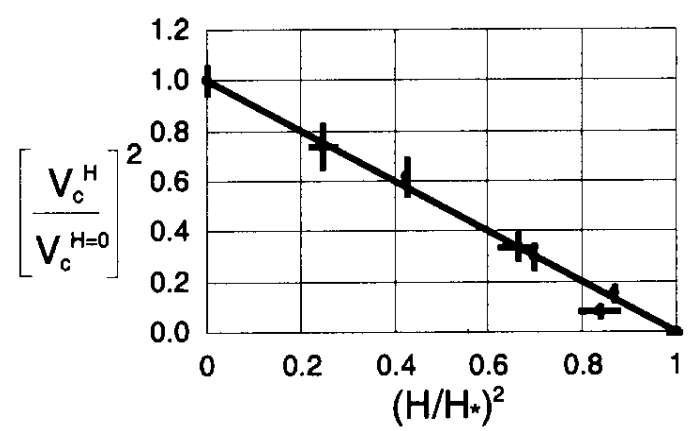

Figure 13. Reduced critical speed $V_{c}^{H} / V_{c}^{H=0}$ at which wave resistance appears in function of the applied reduced magnetic field $H / H_{*}$. The straight line represents the theoretical law given by eq. (13). There is no adjustable parameter. 


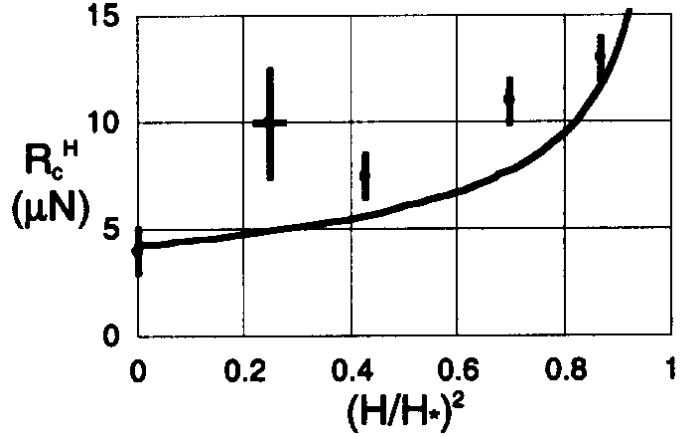

Figure 14. Drag at threshold $R_{c}^{H}$ as a function of the reduced magnetic field $H / H_{*}$. The full line represents the theoretical law as given by eq. (16). There is no adjustable parameter.

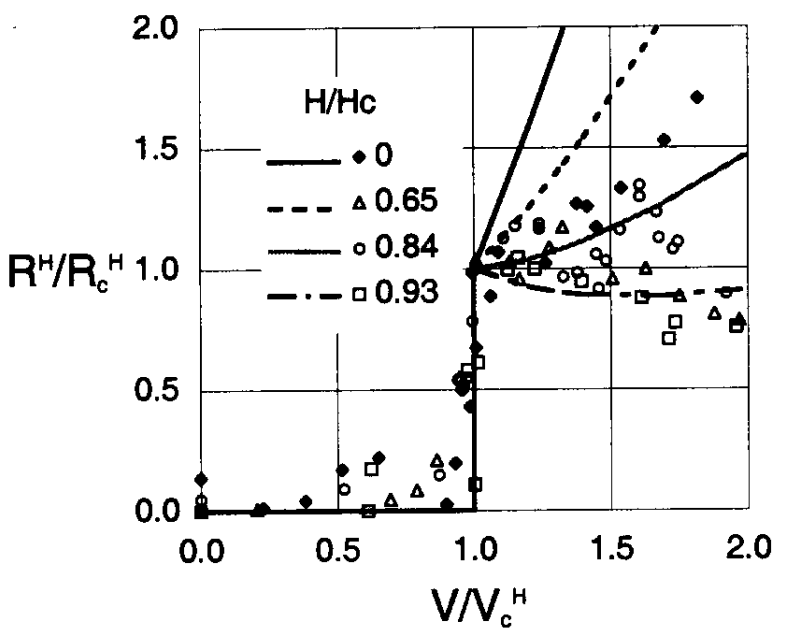

Figure 15. Wave resistance $R^{H}=R_{e x p}^{H}-R_{v i s c}^{H}$ as a function of reduced speed $V / V_{c}^{H}$ for different reduced magnetic fields $H / H_{*}$. The theoretical curves are derived from eq. $(14,15)$. The uppermost curve describes the wave resistance of a regular non-magnetic fluid.

The theoretical expression (13) of $V_{c}^{H}$ (Fig. 13) remarkably fits the data points - note that there are no adjustable parameters. A data point lies outside the curve, but this is probably related to an imperfect magnetic wetting phenomenon. As the magnetic field gets closer to the peak instability threshold value $H_{*}$, the fluid "climbs" onto the wire, producing a much higher viscous drag, a situation which gets away from our inviscid linear theoretical analysis. This also explains the discrepancy in Fig. 14 between experimental and theoretical $R_{c}^{H}$ values. We do not account for the force that the magnetic field is exerting at the meniscus close to the wire. Indeed, the very shape of the meniscus creates a non homogeneous magnetic field which results in a force that sucks the magnetic fluid up and changes the shape of the meniscus. Only advanced numerical simulations would allow to compute the net force added and [19].

In order to compute the wave resistance from the measured drag, as for ordinary fluids, we estimate the viscous drag $R^{\text {drag }}$ using eq. (11). This time, because the immerged length of the wire depends on the magnetic field intensity, each curve at subcritical speed are separately fitted. Fig. 15 presents the results obtained for different magnetic fields in a reduced representation $R^{H} / R_{c}^{H}=f\left(V / V_{c}^{H}\right)$ with $R^{H}=R_{\exp }-R_{v i s c}$. It also gives a comparison to the theoretical predictions of eq. (14). As it was pointed out about regular viscous fluids, the theoretical variations of $R / R_{c}^{H}$ lie above the data points, except for $H \approx H_{*}$. Then the experimental data and the theory are very comparable. The present theoretical description thus gives a correct general trend for the influence of the field on the wave resistance.

\section{Conclusion}

We have designed a shadowgraph experiment in order to measure the dispersion equation of waves at the free surface of a magnetic fluid submitted to a vertical magnetic field. The model includes the specific magnetic behavior of the ferrofluid used, sensible boundary conditions and the effects of limited thickness. The agreement between theory and experience is quite satisfactory except in the region where the dispersion equation is non-monotonic. We have used this knowledge in wave resistance measurements.

A drag discontinuity is always observed at a critical velocity $V_{c}$. Thanks to a magnetic fluid the critical velocity range is experimentally extended. In all cases the measured critical velocities and the critical values of the resistance are in good accordance with the developped model. If an inviscid theory is correct at the threshold, there are some discrepancies for $V>V_{c}$ such as a nonmonotonic behavior of the wave resistance. Viscosity and non linear aspects should be taken into account in further works. Finally, in order to get rid of the viscous drag that is always present in our experiments, another mode of disturbance is envisaged, such as a small magnet placed just above the free surface of a flowing magnetic fluid.

\section{Acknowledgments}

We wish to thank J. Servais and P. Lepert for their technical assistance, O. Cardoso and C. Flament for the help provided in the conception and the tuning of the experimental setup, S. Neveu for providing us with the ferrofluid sample, O. Sandre for granting us access to an accurate tensiometer. Fruitful discussions were made with B. Abou, R. E. Rosensweig and E. Raphaël.

\section{References}

[1] M. D. Cowley. and R. E. Rosensweig, J. Fluid Mech. 30, 671 (1967). 
[2] R. E. Rosensweig, Ferrohydrodynamics (Dover, Mineola, 1997).

[3] S. Chandrasekhar, Hydrodynamic and hydromagnetic stability (Dover, 1981).

[4] J. Weilepp and R. Brand, J. Phys. II 2 ,419 (1996).

[5] Lord Kelvin Proc. R. Soc. London A 42, 80 (1887).

[6] A. A. Kostyukov, Theory of Ship Waves and Wave Resistance (Effective Commun. Inc., Iowa City, 1968).

[7] E. Raphaël and de P.-G. de Gennes, Phys. Rev. E 53, 3448 (1996)

[8] J. Lighthill, Waves ind Fluids, Cambridge University Press, Cambridge, 1996.

[9] R. E. Zelazo and J. R. Melcher, J. Fluid Mech. 39, 1 (1969).

[10] B. Abou, G. Nron de Surgy and J. E. Weisfreid, J. Phys. II France, 7, 1159 (1997).

[11] J. Browaeys, J.-C. Bacri, C. Flament, S. Neveu and R. Perzynski, Eur. Phys. J. B 9, 335 (1999).

[12] F. H. Leblond \& F. Mainardi, Acta Mechanica 68, 203 (1987).
[13] R. Massart, IEEE Trans. Magn. 17, 1247 (1981).

[14] S. Rasenat, G. Hartung, B.L. Winkler and I. Rehberg, Experiments in fluids 7, 412(1989).

[15] The waves are damped but their frequency remains unaffected by viscosity at the first order.

[16] R. W. Fox and A. T. McDonald, Introduction to Fluid Mechanics (John Wiley and Sons, New York, 1994).

[17] D. Richard and E. Raphaël, Europhys. Lett.48, 53 (1999).

[18] Strictly speaking, a real solution exists provided $V / V_{c}>\sqrt{1-H^{2} / H_{*}^{2}} /|\cos \theta|$, where $\theta$ is the angle between the wave vector of the emitted plane wave and the direction of the speed. Waves are therefore not emitted isotropically. The interval of possible angles is given by the preceding equation, or by the boundary of the integral in eq. (14). The absolute minimum value is obtained for $\theta=0$.

[19] A. G. Boudouvis and L. E. Scriven J. Magn. Magn. Mater. 122, 254 (1993). 